\title{
Alopecia areata as a paraneoplastic syndrome of Hodgkin's lymphoma: A case report
}

\author{
JUN GONG ${ }^{1}$ and STEPHEN W. LIM ${ }^{2}$ \\ ${ }^{1}$ Department of Internal Medicine and ${ }^{2}$ Blood and Marrow Transplant Program, \\ Samuel Oschin Comprehensive Cancer Institute, Cedars-Sinai Medical Center, Los Angeles, CA 90048, USA
}

Received November 29, 2013; Accepted April 3, 2014

DOI: $10.3892 / \mathrm{mco} .2014 .274$

\begin{abstract}
Alopecia areata (AA) has been classically associated with several autoimmune disorders. However, AA as a paraneoplastic syndrome of Hodgkin's lymphoma (HL) remains a rare entity and our understanding of this phenomenon is limited to a few case reports. This is the case report of a 46-year-old male patient who was diagnosed with AA several months prior to the onset of B symptoms and the diagnosis of stage IVB classical HL. The patient was subsequently treated with 6 cycles of adriamycin, bleomycin, vinblastine and dacarbazine and experienced a complete response and resolution of his AA. In our case, the onset of AA preceded the onset of systemic manifestations and diagnosis of HL, whereas in other cases AA was shown to occur concurrently with the clinical manifestations of HL. In all the cases, however, treatment of the HL subsequently led to resolution of the AA. The present case report highlights the significance of AA as a herald of underlying malignancy, although AA in classical HL remains poorly characterized in the literature.
\end{abstract}

\section{Introduction}

Alopecia areata (AA) has been classically associated with several autoimmune disorders (1). Along with follicular mucinosis, AA has also been historically documented in panniculitis-like T-cell lymphoma, primary cutaneous follicle center lymphoma, cutaneous T-cell lymphomas, including mycosis fungoides and Sézary syndrome, as well as other non-Hodgkin's lymphomas (NHLs) (2-5). AA, however, as a paraneoplastic syndrome of Hodgkin's lymphoma (HL), remains limited to a few case studies, although its occurrence in HL was anecdotally referenced in the early 1900s (6-9). The etiology of AA as a paraneoplastic syndrome

Correspondence to: Dr Jun Gong, Department of Internal Medicine, Cedars-Sinai Medical Center, 8700 Beverly Blvd, room 5512, Los Angeles, CA 90048, USA

E-mail: jun.gong@cshs.org

Key words: alopecia areata, paraneoplastic syndrome, Hodgkin's lymphoma of HL remains unknown, although it was considered to be a T-lymphocyte-mediated phenomenon involving production of cytokines, initiation of the inflammatory cascade and genetic predisposition $(1,4,8,10)$.

\section{Case report}

A 46-year-old male with a history of hypertension presented to the outpatient clinic with complaints of patchy hair loss on his mustache and beard for 5 months. His review of systems was otherwise unremarkable. The physical examination revealed at least 8 prominent regions of patchy, non-tender, non-erythematous hair loss, without scarring of the underlying skin, in the patient's beard and mustache area, with the largest lesion located at mid-chin. The patient was treated for AA with a low-potency topical corticosteroid and subsequent intralesional injection with $10 \mathrm{mg}$ triamcinolone acetonide, with eventual improvement. Approximately 8 months after the initial diagnosis of AA, the patient presented to the emergency room with intermittent fevers, night sweats, weight loss and abdominal pain. The assessment of the vital signs revealed a temperature of $101.5^{\circ} \mathrm{F}$ and a heart rate of $122 \mathrm{bpm}$. On physical examination there was significant tachycardia, abdominal obesity and 3 regions of patchy hair loss in the mustache and beard area, with the largest lesion $(\sim 3 \times 4 \mathrm{~cm})$ located at midchin, with characteristics as previously described (Fig. 1).

The laboratory examinations revealed notable pancytopenia, elevated erythrocyte sedimentation rate, normal lactate dehydrogenase levels, normal thyroid function tests and a negative antinuclear antibody (ANA) titer. The computed tomography (CT) of the chest, abdomen and pelvis and magnetic resonance imaging of the abdomen and pelvis demonstrated splenomegaly with multiple nodular hypodensities scattered throughout the spleen, innumerable retroperitoneal and periportal lymphadenopathy and left axillary lymphadenopathy, raising suspicion of lymphoma (Fig. 2). A laparoscopic splenectomy was performed and the histopathological examination revealed the presence of large atypical cells resembling Reed-Sternberg cells admixed in a background of lymphocytes and fibrosis, with staining characteristics consistent with those of classical HL of the nodular sclerosis subtype (positive for CD15, CD30, fascin, Epstein-Barr virus latent membrane protein-1 and CD20). The bone marrow biopsy also revealed the presence of large atypical cells with prominent nucleoli 


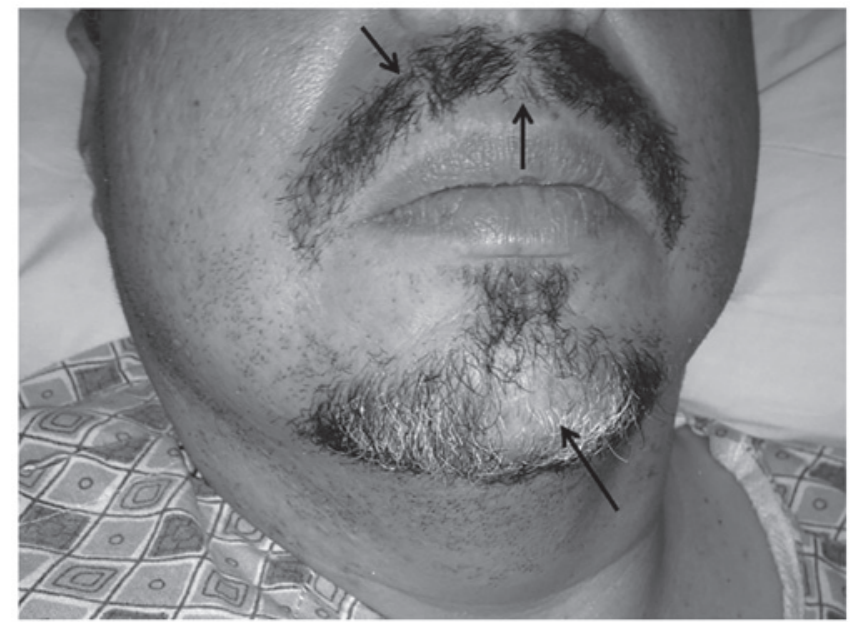

Figure 1. The physical examination revealed 3 regions of patchy, non-tender, non-erythematous hair loss (arrows), without scarring of the underlying skin, in the mustache and beard area, with the largest lesion $(\sim 3 \times 4 \mathrm{~cm})$ located at mid-chin.

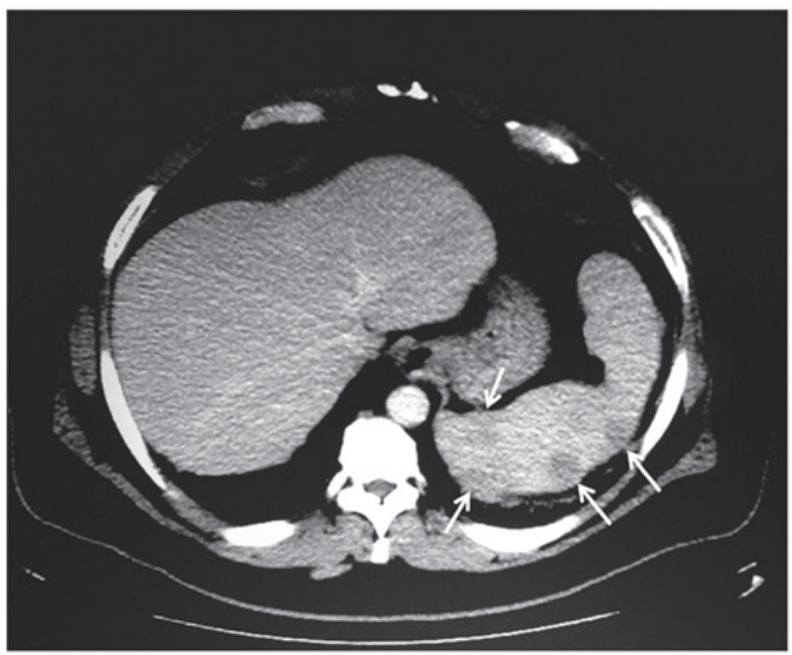

Figure 2. Computed tomography scan of the chest, abdomen and pelvis revealed splenomegaly with multiple nodular hypodensities scattered throughout the spleen, raising suspicion of lymphoma.

and staining characteristics similar to those mentioned above. The patient subsequently underwent 6 cycles of adriamycin, bleomycin, vinblastine and dacarbazine (ABVD) for stage IVB HL. At the time of this study, a surveillance positron emission tomography-CT scan revealed no evidence of recurrence and the patient's AA had virtually resolved.

\section{Discussion}

AA is a benign condition characterized by sudden, patchy, non-scarring hair loss, possibly due to T-lymphocyte-mediated destruction of hair follicles via cytokine production and initiation of the inflammatory cascade (1). AA has been associated with autoimmune disorders, such as vitiligo and thyroid disease; however, the association of AA with diabetes, lupus erythematosus, pernicious anemia, myasthenia gravis, rheumatoid arthritis, polymyalgia rheumatica, ulcerative colitis and lichen planus have also been documented (1). With respect to lymphomas, AA and follicular mucinosis or alopecia mucinosa, a condition commonly characterized by erythematous plaques or coalescing follicular papules with scarring and hair loss, have been historically documented in cutaneous T-cell lymphomas, including mycosis fungoides and Sézary syndrome (2-4).

A previous epidemiological study reported that, out of 33,271 patients with NHL and 9,474 with multiple myeloma, 19 and 10 patients, respectively, had associated AA (5). The association between AA and HL, or as then termed, Hodgkin's disease, was anecdotally referenced in the 1920s, or even earlier (6). It was also reported that other dermatological conditions, including pruritic, urticarial, hemorrhagic, exfoliative, pigmented and bullous eruptions, were associated with HL (6). However, to the best of our knowledge, the incidence of AA in association with HL is exceedingly rare. In fact, out of 1,155 cases of HL, there were $<5$ cases of associated AA at the time of a previously published review (5) and our knowledge of AA as a paraneoplastic phenomenon of HL is limited to a few case studies (7-9).

The clinical presentations of AA in such cases were characterized by patchy regions of hair loss to complete hair loss on the scalp (alopecia totalis) (7-9). In one case, the manifestations of AA preceded the majority of the systemic manifestations of HL by several months (7), whereas the other cases presented with concurrent manifestations of AA and HL $(8,9)$. Two of the cases occurred in young females (aged 17 and 30 years), whereas one case occurred in a 43-year-old male (7-9). Other clinical manifestations included generalized pruritus, diffuse skin scaling, diffuse skin hyperpigmentation, fevers, weight loss, anorexia, fatigue, hepatomegaly, back pain, scoliosis and painless cervical, axillary and inguinal lymphadenopathy (7-9). The thyroid function tests, serum cortisol, serum adrenocorticotropic hormone, C3 and C4 levels and ANA titers were notably negative $(8,9)$. All the reported cases had advanced disease (Ann Arbor stage IIIB or IVB) and received chemotherapy with either ABVD, mustargen, oncovin (vincristine), procarbazine and prednisone (MOPP) with ABV, or oncovin, prednisone, procarbazine and doxorubicin (OPPA) with cyclophosphamide, oncovin, prednisone and procarbazine (COPP) (7-9). Following chemotherapy, the AA resolved as early as after 1 cycle of ABVD to as late as 12 months following completion of OPPA and COPP (7-9).

The etiology of AA in classical HL has not been fully elucidated. It has been suggested that AA may be the result of direct infiltration and destruction of hair follicles by the disease or, more commonly, a paraneoplastic phenomenon (7). The mechanism underlying this paraneoplastic process has been putatively attributed to impaired cellular immune responses or the anergy seen in HL, which correlate with the findings of reduced numbers of circulating T-lymphocytes (in particular, $\mathrm{CD}^{+}$suppressor T-lymphocytes) in AA $(1,8)$. Alternatively, other studies suggested that the induction of major histocompatibility complex class I and II expression on hair follicles may lead to the activation of $\mathrm{CD}^{+} \mathrm{T}$-lymphocytes towards hair follicle melanocytes and recruitment of $\mathrm{CD}^{+} \mathrm{T}$-lymphocytes that further attack the hair follicles, a process that may be perpetuated and amplified by the clonal T-cell proliferation seen in lymphomas (4). There is also a genetic predisposition 
contributing to such phenomena, given the numerous human leukocyte antigen subtypes shared by AA and HL $(1,10)$. The resolution of AA observed with the remission of HL in our patient and other case reports reaffirms the close association between these two conditions. However, the phenomenon of AA as a paraneoplastic manifestation of HL remains poorly characterized in the literature. This case report serves to highlight the significance of AA as a herald for possible underlying malignancies and the need for greater awareness of AA and its implications on the prognosis of HL.

\section{References}

1. Madani S and Shapiro J: Alopecia areata update. J Am Acad Dermatol 42: 549-566, 2000.

2. Bonta MD, Tannous ZS, Demierre M, Gonzalez E, Harris NL and Duncan LM: Rapidly progressing mycosis fungoides presenting as follicular mucinosis. J Am Acad Dermatol 43: 635-640, 2000.
3. Török L,Gurbity TP, Kirschner Á and Krenács L: Panniculitis-like T-cell lymphoma clinically manifested as alopecia. Br J Dermatol 147: 785-788.

4. Richmond HM, Lozano A, Jones D and Duvic M: Primary cutaneous follicle center lymphoma associated with alopecia areata. Clin Lymphoma Myeloma 8: 121-124, 2008.

5. Anderson LA, Gadalla S, Morton LM, et al: Population-based study of autoimmune conditions and the risk of specific lymphoid malignancies. Int J Cancer 125: 398-405, 2009.

6. Fox H: Lymphogranulomatosis of the skin in Hodgkin's disease. Arch Derm Syphilol 2: 578-593, 1920.

7. Garg S, Mishra S, Tondon R and Tripathi K: Hodgkin's lymphoma presenting as alopecia. Int J Trichology 4: 169-171, 2012.

8. Mlczoch L, Attarbaschi A, Dworzak M, Gadner H and Mann G: Alopecia areata and multifocal bone involvement in a young adult with Hodgkin's disease. Leuk Lymphoma 46: 623-627, 2005.

9. Chan PD, Berk MA, Kucuk O and Singh S: Simultaneously occurring alopecia areata and Hodgkin's lymphoma: complete remission of both diseases with MOPP/ABV chemotherapy. Med Pediatr Oncol 20: 345-348, 1992.

10. Mani $\mathrm{H}$ and Jaffe ES: Hodgkin lymphoma: an update on its biology with newer insights into classification. Clin Lymphoma Myeloma 9: 206-216, 2009. 